\title{
Above Threshold Estimation of Alpha (Henry) Parameter in Stripe Lasers Using Near- and Far-Field Intensity Measurements
}

\author{
Roberto Pagano, Student Member, IEEE, Jayanta Mukherjee, Pawel Sajewicz, and Brian Corbett
}

\begin{abstract}
Henry parameter, $\alpha_{H}$, is a key wavelength and carrier density dependent parameter which is central in determining the dynamic behavior of semiconductor lasers. While it may be extracted from below threshold measurements, its characteristics are of great interest above threshold where the lasers operate. We show that $\alpha_{H}$ along with the lateral gain profile may be determined above threshold in stripe laser structures through recovery of the lateral near-field phase profile. The lateral phase profile of the wave-front at the facet is calculated using measured near- and far-field intensities of the laser based on a model under scalar Helmholtz equation formulation. It is shown that $\alpha_{H}$ attains its maximum value under or in the proximity of the center of the pumped stripe. We apply the method to AlGaAs $(\lambda=776 \mathrm{~nm})$ lasers with stripe widths ranging from $6-50 \mu \mathrm{m}$ and with two levels of lateral current spreading achieved by having two different $p$-side cladding layer thicknesses. This permits comprehensive interpretation and analysis of the measured nearand far-field characteristics.
\end{abstract}

Index Terms-Alpha parameter, anti-guiding, broad area lasers, gain guiding, gain profile, Henry parameter, phase extraction, stripe lasers.

\section{INTRODUCTION}

$\mathbf{H}$ ENRY alpha parameter $\left(\alpha_{H}\right)$ which connects the differential change of refractive index with the corresponding change in gain has been widely discussed since its introduction into the field of semiconductor lasers [1]. An early review on this topic can be found in [2]. This parameter is defined as:

$$
\alpha_{H}=-\frac{4 \pi}{\lambda} \frac{d n / d N}{d g / d N},
$$

where $n$ is the refractive index and $g$ the modal gain, both functions of carrier density $N$, which in turn is a spatially varying function at a fixed temperature. The derivative in Eq. (1) has to be taken as a function of the carrier density only and at the gain spectral peak. For our purpose we will consider only a lateral spatial dependence (i.e. on the $\mathrm{x}$-coordinate)

Manuscript received July 20, 2010; revised October 1, 2010; accepted November 1, 2010. Date of current version March 9, 2011. This work was supported in part by Enterprise Ireland under Contract CFTD/2004/314 and the European Commission within the project Brighter.EU under Contract 035266.

R. Pagano, P. Sajewicz, and B. Corbett are with the Tyndall National Institute, University College Cork, Cork, Ireland (e-mail: roberto.pagano@ tyndall.ie; pawel.sajewicz75@gmail.com; brian.corbett@tyndall.ie).

J. Mukherjee is with the Photonics Group, University of Surrey, Guildford, Surrey GU2 7XH, U.K. (e-mail: j.mukherjee@surrey.ac.uk). assuming a reference system as depicted in Fig. 1 (a). The negative sign in Eq. (1) is introduced to make $\alpha_{H}$ a positive quantity as the refractive index variation with the carrier density, $\mathrm{dn} / \mathrm{dN}$, is negative. By operating the laser with pulse widths $<500 \mathrm{~ns}$, as done in our experiments, the Joule heating of the lattice becomes negligible and $\alpha_{H}$ becomes a function of only space and carrier density. It is not correct, then, to assume the alpha parameter as a constant independent of the device geometry, an assumption often made for its experimental determination [3], [4] as well for the purpose of modeling (see for example [5], [6]). This parameter is responsible for the frequency chirp of diode lasers under direct modulation (spectral influence) and on the formation of filaments in broad area diode lasers (spatial influence). The higher is its value the higher the chirp or the number of filaments. Filamentation is a phenomenon that takes place in stripe lasers [7], [8], [9] which, as shown by Thompson and Kirkby [10], [11], occur when the gain and the real part of the dielectric constant have a spatial offset in their relative peaks.

It is important to underline the difference between $\alpha_{H}$ as measured below and above laser threshold. Below threshold all the devices made with the same material present the same value of $\alpha_{H}$, independently of the device geometry. $\alpha_{H}$ is generally calculated by employing the method proposed by Hakki and Paoli [12], which is based on tracking the shift of the Fabry-Perót longitudinal cavity mode peaks with the current. The parameter is then just a function of the frequency spectrum (energy) and no information about the lateral dependence of the gain and the refractive index is included as, below threshold, in a uniformly pumped region of the device, the gain and the refractive index are considered to be uniformly distributed and weakly coupled. Below threshold, then, the alpha parameter of the material can be measured. Above threshold, instead, as the gain and refractive index spatial distributions can be no longer considered uniform and, especially for broad area lasers, the role played by the device geometry becomes relevant. The alpha parameter measured is a function of the space and its value both influences and is influenced by the spatial distribution of optical field. Above threshold then the alpha parameter of the device is measured. We will discuss below, how the spatial profile of $\alpha_{H}$ influences the performance of stripe lasers and how to recover it easily above threshold where the Hakki-Paoli method cannot be applied.

The behavior of stripe lasers is strongly influenced by the carrier injection. The increase in carrier concentration lowers 


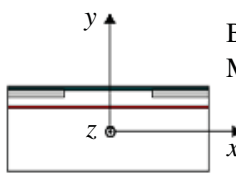

(a)

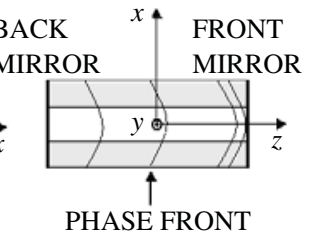

(b) (c)

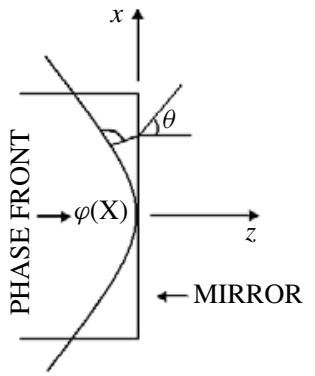

Fig. 1. (a) Front facet of the laser device together with the relative coordinate system. (b) Laser cavity as seen from the top with the lateral profile of the phase fronts propagating along z. (c) Blow-up of the lateral phase profile at the laser facet and corresponding far-field radiation angle, $\theta$.

the refractive index inducing carrier anti-guiding [10]. As long as the guiding due to the gain is sufficient to overcome the defocusing effect of the index decrease, the laser operates in the gain-guiding mode. However, the competition between gain-guiding and anti-guiding may lead to instabilities in the laser performance [8]. Stimulated emission depletes the carriers (spatial hole burning), increasing the local refractive index. This effect, known as self-focusing [10], [13], confines the light further increasing the local field intensity until the gain saturates. A combination of these phenomena, i.e. carrier induced anti-guiding, self-focusing and spatial hole burning, is responsible for the formation of the near field of the stripe laser.

In this paper we present a new method to extract the Henry parameter from stripe lasers ( $6 \mu \mathrm{m}-50 \mu \mathrm{m}$, stripe widths) by using the measured near- and far-field intensities. The lasers have no structural positive refractive index step in the lateral direction or, by operation in pulsed mode, any thermally induced positive local increase in the refractive index. A simple model, based on the scalar Helmholtz equation, provides a way to extract the near-field lateral phase front from which above threshold lateral gain and lateral $\alpha_{H}$ profiles are deduced. The results obtained are used to explain the specific behavior of the lasers as a function of stripe width.

The paper is organized as follows: Section II describes the model used to extract the lateral phase profile from the measured near- and far-fields. Section III describes the device structures investigated and the experimental measurements performed while section IV deals with the analysis of the experimental results. The conclusions are presented in section V.

\section{MOdEL FOR EXTRACTION OF LATERAL Phase PROFILE}

\section{A. Phase Profile Extraction Model}

The far-field of a laser can be approximated by the Fourier transform of the near-field when the far-field is measured beyond than the Rayleigh range. Calculation of the far-field requires the knowledge of the near-field amplitude as well as the phase profile. However, as optical detectors measure only the intensities of near- and far-fields, information about the phase profile is lost. One can recover the phase information using a sophisticated technique, as shown by Braun et al. [14], however, as we show below, for a highly astigmatic laser beam, the lateral near-field phase front can be recovered with a good approximation by applying the stationary phase approximation [22]. In fact, the phase front of the electric field of a highly astigmatic beam is rapidly-varying, fulfilling the condition to apply the stationary phase approximation. A laser without an inbuilt (due to waveguide geometry) or thermally-induced positive step in the refractive index along the lateral direction is highly astigmatic and has a convex lateral field phase front, as the one shown in Fig. 1 (c), where a schematic of the gain guided stripe laser with beam propagation along the $\mathrm{z}$-axis is represented. Along the transverse direction the phase front is flat because the built-in refractive index profile is dominant on any possible perturbation due to the carrier density. The complex lateral near-field amplitude at the laser facet, $E_{N F}(x)$, can be described by the solution to the scalar Helmholtz equation based on effective index method approximation [6], [8], [13]:

$E_{N F}^{\prime \prime}(x)=k_{0} n_{b}\left\{\left[i+\alpha_{H}(x)\right] \Gamma_{y} g(x)-i \alpha_{L O S S}\right\} E_{N F}(x)$,

where $k_{0}=2 \pi / \lambda$ the wave number and $\lambda$ the peak wavelength, $n_{b}$ is the background refractive index of the semiconductor, $\mathrm{i}$ is the imaginary unit, $\alpha_{H}(x)$ is the Henry parameter, $\Gamma_{y}$ is the transverse confinement factor, $g(x)$ is the lateral gain profile, $\alpha_{\text {Loss }}$ represents the total losses (i.e. mirror and internal loss). Equation (2) is valid just above threshold and is applicable if the laser operates in a single longitudinal mode (lasing mode). In a broad area laser all laterals modes follow the behavior of the modes corresponding to the longitudinal mode under the peak-gain. Thus, for modeling and analyzing lateral modal effects in broad area lasers a single longitudinal mode model is sufficient.

Following Petermann's approach [15], which is based on the stationary phase approximation, the lateral near-field can be written as:

$$
E_{N F}(x)=\exp [-\gamma(x)+i \phi(x)]
$$

where

$$
\gamma(x)=\ln \left[I_{N F}(x)\right] / 2 .
$$

$I_{N F}(x)$ is the lateral near-field intensity distribution normalized to its maximum intensity and $\phi(x)$ is the lateral near-field phase profile.

Within the large astigmatism hypothesis the far-field intensity is not given by a superposition of interfering parts of the near-field. Rather, the far-field angular distributions are made up from the different parts of the near-field, radiating into separate angles, $\theta$. Applying the stationary phase approximation it can be shown that:

$$
\phi^{\prime}(x)=-k_{0}\left[\Theta_{N}(x)+\Theta_{C}\right] \theta_{\max },
$$

where $\Theta_{N}(x)\left(-1 \leq \Theta_{N}(x) \leq 1\right)$ is the normalized farfield angle (a function of the lateral position), meaning that all the energy contained in the far-field is stored within this range while $\theta_{\max }$ is the maximum far-field angle and $\Theta_{C}$ an integration constant which is set equal to zero to centre the farfield at the angle $0^{\circ}$. The integral of the normalized intensity of 
the near-field is equal to the integral of the normalized far-field intensity,

$$
\int_{-X}^{X} I_{N F(n o r m)}(x) d x=\int_{-\Theta_{N} \min }^{\Theta_{N \max }} I_{F F(n o r m)}\left(\Theta_{N}\right) d \Theta_{N},
$$

where $I_{N F(\text { norm })}$ and $I_{F F(n o r m)}$ are respectively the normalized lateral near- and far- field intensities. Equation (6) can be also expressed in its differential form as:

$$
\Theta_{N}^{\prime}(x)=I_{N F(\text { norm })} / I_{F F(\text { norm })} .
$$

Therefore, using (5) and (7) one can recover the lateral phase profile provided the measured near- and far-field intensities are measured.

\section{B. Gain and Henry Parameter Profiles}

If the lateral phase profile is extracted exactly then an estimation of the lateral Henry parameter and gain profile above threshold is possible. Substituting (3) in (2) and extracting the imaginary part gives the lateral gain profile,

$$
\Gamma_{y} g(x)=\left[\phi^{\prime \prime}(x)-2 \gamma^{\prime}(x) \phi^{\prime}(x)+k_{0} n_{b} \alpha_{L O S S}\right] / k_{0} n_{b}
$$

Taking the ratio between the real and the imaginary part we obtain,

$$
\alpha_{H}(x)=\frac{-\gamma^{\prime}(x)+\left[\gamma^{\prime}(x)\right]^{2}-\left[\phi^{\prime}(x)\right]^{2}}{\phi^{\prime \prime}(x)-2 \gamma^{\prime}(x) \phi^{\prime}(x)+k_{0} n_{b} \alpha_{L O S S}}+\alpha_{H \infty} .
$$

This equation shows that the $\alpha_{H}$ parameter is not a constant but has a lateral profile with a magnitude that is reduced when the total losses increase. A constant $\alpha_{H \infty}$ is added in (9) so that $\alpha_{H}$ equal to zero outside the laser stripe (a spatial boundary condition), where all the near- field intensity dependent terms and the second derivative of the phase profile are null. The value of $\alpha_{H \infty}$ is the one that $\alpha_{H}$ would have if it was just a a spatially-independent parameter. It is evaluated as,

$$
\alpha_{H \infty}=\left[\phi^{\prime}(x)\right]^{2} / k_{0} n_{b} \alpha_{L O S S} .
$$

Using Eq. (5), $\alpha_{H \infty}$ can be expressed as:

$$
\alpha_{H \infty}=k_{0} \theta_{\max }^{2} / n_{b} \alpha_{L O S S}
$$

This relation shows that devices characterized by a smaller Henry parameter will diverge with a smaller far-field maximum angle if the mirror losses are considered as a constant. The expression of Eq. (11) is physically identical to the Eq. (20) in reference [6]. It's worth to notice that the insertion of the constant $\alpha_{H \infty}$ doesn't modify Eq. (2).

An exact evaluation of the lateral profile of $\alpha_{H}$ is dependent on the degree of accuracy with which the phase profile is calculated which in turn is related to the accuracy of the measurement of maximum far-field intensity angle, $\theta_{\max }$. The method proposed is very sensitive to the accuracy of the measured intensities and the noise is a potential source of significant errors in the estimation of the phase profile. Hence, particular care is required in the measurements of the near- and far-field intensities for our method to yield accurate results.

In this work an iterative procedure has been applied in order to optimize the extraction of the phase profile. The procedure

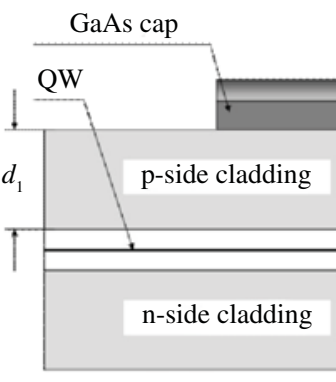

(a)

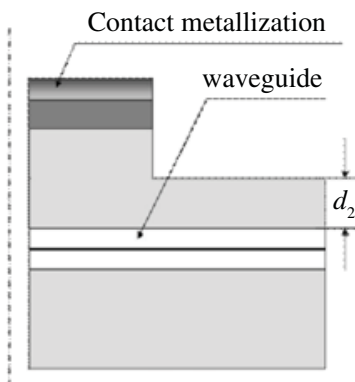

(b)
Fig. 2. Schematic representation of the two device structures investigated: (a) Structure A where only the GaAs cap layer is etched. (b) Structure B where the residual p-side cladding layer thickness was reduced to $0.2 \mu \mathrm{m}$.

is as follows: (1) an initial phase profile is calculated from the ratio between the measured near- and far-field intensities, (2) the far-field intensity is calculated and compared with that measured and finally, (3) the phase profile is refined until the best match between the calculated and experimental far-field intensity is achieved. A good match with the experimental farfield intensity data was obtained by applying this algorithm.

\section{EXPERIMENTAL RESULTS}

\section{A. Devices and Setup}

Gain guided stripe lasers with two levels of current spreading were investigated. The devices used a $776 \mathrm{~nm}$ emitting epitaxial structure consisting of a $80 \mathrm{~nm}$ thick $\mathrm{Al}_{0.3} \mathrm{Ga}_{0.7}$ As waveguide embedded between a top and bottom $1.5 \mu \mathrm{m}$ thick $\mathrm{Al}_{0.55} \mathrm{Ga}_{0.45} \mathrm{As}$ cladding layers. The active region contains three $5.5 \mathrm{~nm}$ thick $\mathrm{Al}_{0.09} \mathrm{Ga}_{0.91}$ As quantum wells with $6 \mathrm{~nm}$ thick $\mathrm{Al}_{0.3} \mathrm{Ga}_{0.7} \mathrm{As}$ barriers. Oxide isolated, stripe contact lasers with widths, W, between $6 \mu \mathrm{m}$ and $50 \mu \mathrm{m}$ were fabricated. Devices with two different residual thicknesses of the p-side cladding layer outside the contact stripe were fabricated. The first set where only the GaAs p-type contact layer is etched are identified as structure A (Fig. 2 (a)), while devices where the $\mathrm{Al}_{0.55} \mathrm{Ga}_{0.45}$ As layer is substantially etched leaving a $0.2 \mu \mathrm{m}$ thick residual p-side cladding layer are identified as structure B (Fig. 2 (b)). The etching of the p-side cladding layer from $1.5 \mu \mathrm{m}$ to $0.2 \mu \mathrm{m}$ increases sheet resistance of that layer from $600 \Omega$ to $4500 \Omega$, and significantly decreases the lateral current spreading [17]. In both cases any built-in step in the lateral refractive index is minimal. The facets of the lasers were left uncoated and devices with a cavity length, $\mathrm{L}=1000 \mu \mathrm{m}$ are used.

The lasers were operated with 500 ns long current pulses (with $0.1 \%$ duty cycle) in order to eliminate Joule heating while the mount temperature was fixed to $15{ }^{\circ} \mathrm{C}$. The LightCurrent (L-I) characteristics were measured which showed a rapid increase of the nominal (i.e. evaluated as the ratio between the threshold current and the area of the stripe), threshold current density $\mathrm{J}_{\text {th }}$, especially for stripe widths less than $20 \mu \mathrm{m}$ (Fig. 3). This is as a result of the significant current spreading in the p-side cladding layer [16].

The near field intensity pattern was measured laterally by re-imaging the light at the facet with a single lens onto a linear 


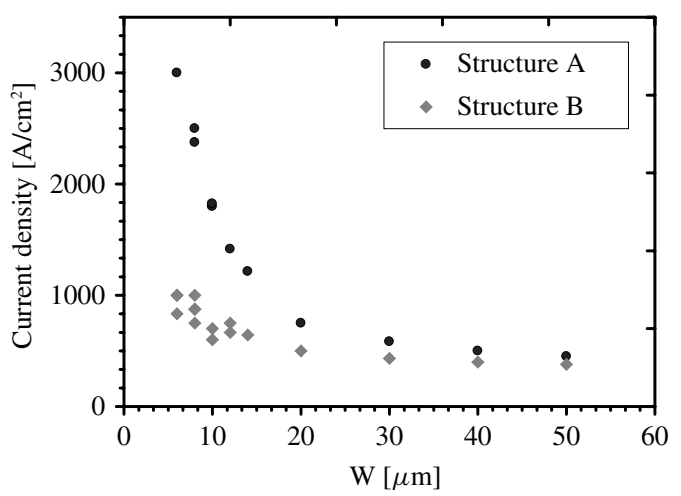

Fig. 3. Nominal (i.e., evaluated as the ratio between the threshold current and the area of the stripe) threshold current densities, $\mathrm{J}_{\text {th }}$, measured from structure A (dots) and structure B (squares) as function of the stripe width, W.

detector. The far-field intensities were collected with the same detector without any lenses and corrected for obliquity.

\section{B. Structure A Lasers}

In Fig. 4 (a), (c) and (e), we show the measured near-field intensities (black curves) and the corresponding calculated gain profiles (grey curves) for the shallow etched (structure A) lasers. The characteristics are shown for $6 \mu \mathrm{m}, 14 \mu \mathrm{m}$ and $50 \mu \mathrm{m}$ wide stripes measured at 1.5 times threshold current. For stripe widths between $6 \mu \mathrm{m}$ to $10 \mu \mathrm{m}$ (the $6 \mu \mathrm{m}$ case shown in Fig 4(a)) the lateral near-field intensity is characteristically double lobed with a dip in the intensity at the centre and with a lateral extent many times the stripe contact width. The dip in the intensity becomes deeper with the increasing current while the full width at half maximum (FWHM) of the near field increases. The far-field intensity (see Fig. 4 (b)) is double peaked and the angular separation of two peaks remains constant while the dip between becomes deeper when the current increases. By blocking part of the near-field intensity, so that only one lobe is left and measuring the corresponding far-field intensity, it can be shown that when the left lobe of the near-field intensity is blocked the left lobe in the far-field intensity disappears while there is no change in the intensity of the remaining part of the near-far-field intensity profile as observed by Lang [6] and Houlihan [17]. This indicates that each lasing element in the near-field radiates into a different far-field angle and so that the phase profile is convex, as already discussed in section II.

For lasers with a stripe widths between $12 \mu \mathrm{m}$ to $20 \mu \mathrm{m}$ (the $14 \mu \mathrm{m}$ case is shown in Fig. 4 (d)) the nominal threshold current density is considerably lower than for the $6 \mu \mathrm{m}$ wide stripe $\left(1200 \mathrm{~A} / \mathrm{cm}^{2}\right.$ compared with $\left.3000 \mathrm{~A} / \mathrm{cm}^{2}\right)$, but kinks are measured in the L-I characteristic (not shown). The nearfield intensity is broader than the effective contact width and is strongly asymmetric. The asymmetry in the near-field intensity starts just above the threshold and becomes stronger with increasing current.

For lasers with a stripe width $>20 \mu \mathrm{m}$ (the $50 \mu \mathrm{m}$ case is shown in Fig. 4 (e)) the near-field intensity evolves from a single- to a multi-lobe as is typical for broad area, multimode diode lasers [17]. The far-field intensity, shown in Fig. 4
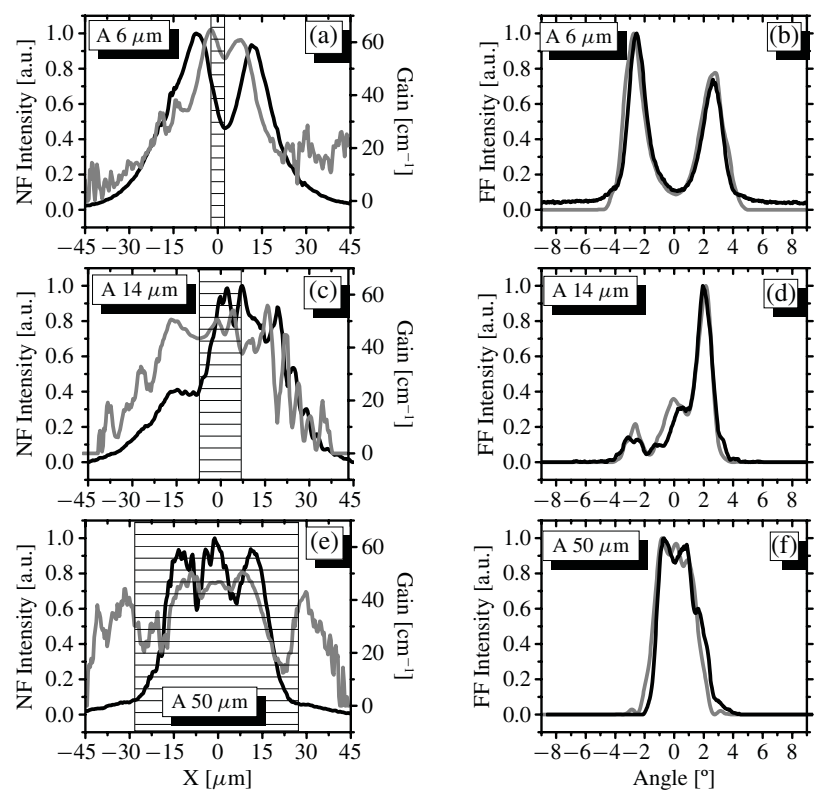

Fig. 4. Measured lateral near-field intensities (black curves) along with the extracted gain profiles (grey curves) [(a), (c), and (e)] and the corresponding measured far-field intensities (black curves) together with the calculated farfield intensities (grey curves) [(b), (d), and (f)] at $\mathrm{I}=1.5 \mathrm{I}_{\text {th }}$ for lasers with structure A. The stripe widths are $6 \mu \mathrm{m}$ (top), $14 \mu \mathrm{m}$ (middle) and $50 \mu \mathrm{m}$ (bottom). The shaded areas indicate the stripe width.

(f), is mainly single-lobed with additional features, recalling the typical behavior of high order modes in gain guided structures.

\section{Structure B Lasers}

Significant differences are observed in the lateral optical field behavior in stripe lasers with reduced current spreading (structure B) compared with those of structure A. With reference to Fig. 5, the near-field intensity for $6 \mu \mathrm{m}-10 \mu \mathrm{m}$ wide stripe lasers is much narrower (the $6 \mu \mathrm{m}$ case is shown in Fig. 5 (a))), single-lobed with a lateral shoulder. The peak of the lobe is inside the contact stripe but is not centered. The near-field intensities of $12 \mu \mathrm{m}-20 \mu \mathrm{m}$ wide stripes are asymmetric but, unlike the corresponding structure A devices (see Fig. 4 (a), (c) and (e)), the far-field intensities are double lobed and slightly asymmetric (the $14 \mu \mathrm{m}$ case is shown in Fig. 5 (c)). Lasers with stripe widths wider than $20 \mu \mathrm{m}$ (the $50 \mu \mathrm{m}$ case is shown in Fig. 5(e)) show a near-field intensity profile with the characteristics lateral "bat ears" [19] that contain a first order mode-like near-field pattern between them. The far-field intensity is multi-peaked with a central main lobe surrounded at both sides by sharply peaked lobes.

\section{ANALYSIS OF EXPERIMENTAL RESULTS}

\section{A. Near- and Far-Field Intensities for Structure A}

The method introduced in section II was applied to the $6 \mu \mathrm{m}, 14 \mu \mathrm{m}$ and $50 \mu \mathrm{m}$ wide stripe laser structures. The calculations of the phase, gain and $\alpha_{H}$ lateral profiles were made with $\lambda=776 \mathrm{~nm}, \alpha_{\text {Loss }}=44 \mathrm{~cm}^{-1}, \mathrm{n}_{\mathrm{b}}=3.6335$. For the devices with large current spreading (structure A) the match between the measured and calculated far-field intensities 

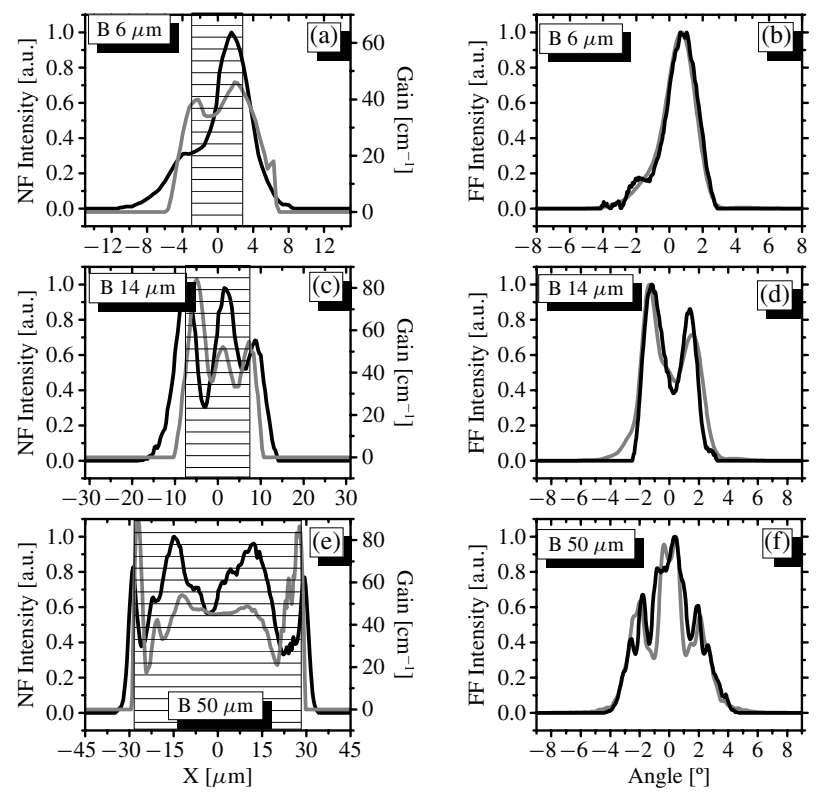

Fig. 5. Measured lateral near-field intensities (black curves) along with the extracted gain profiles (grey curves) [(a), (c), and (e)] and the corresponding measured far-field intensities (black curves) together with the calculated farfield intensities (grey curves) [(b), (d), and (f)] kat $\mathrm{I}=1.5 \mathrm{I}_{\text {th }}$ for lasers with structure B. The stripe widths are $6 \mu \mathrm{m}$ (top), $14 \mu \mathrm{m}$ (middle) and $50 \mu \mathrm{m}$ (bottom). The shaded areas indicate the stripe width.

are shown in Fig. 4 (b), (d) and (f). The calculated lateral gain profiles for these devices are broader than those corresponding to structure B devices, shown in Fig. 5 (a), (c) and (e)), due to an enhanced lateral current spreading in the structure A devices. The plots of Fig. 4 (a), (c) and (e) indicate that the interaction between the lateral gain and the photon density is decided by the extent of current spreading. For the narrowest stripe widths $(<10 \mu \mathrm{m})$ the optical field diffracts within a relatively narrow pumped region leaving the gain relatively high at the center of the stripe, which via carrier induced index anti-guiding creates the resulting near-field (see Fig. 4 (a)). This phase-amplitude coupling, quantified by $\alpha_{H}$, initiates the lasing action at the edges of the pumped region, leading to the formation of a twin-lobed near field intensity which cannot be considered as a first order mode of a gain guided device.

The wide-stripe lasers (here taken from $20 \mu \mathrm{m}$ to $50 \mu \mathrm{m}$ ) are characterized by a very broad gain profile (see Fig. 4 (e)). The refractive index, even if lower than that of the un-pumped region, has a broad profile with a smooth evolution between the pumped and un-pumped regions due to the large current spreading. In this situation the field depletes the carriers, locally increasing the refractive index. This generates a local waveguide sufficient to support the formation of what seem to be higher order mode. This mode-like near-field is sustained by the broad gain profile (Fig. 4(e)), so its nature is gain guided. The near-field width is less than $50 \mu \mathrm{m}$ because the lateral refractive index profile has two strong lobes just inside the stripe corresponding to the weak regions in the lateral gain profile (Fig. 4 (e)). Since the carrier density is low in these regions, the lateral mode (comprising of laterally traveling waves [20]) gets reflected at these lateral relatively high index steps and gets confined within the stripe.
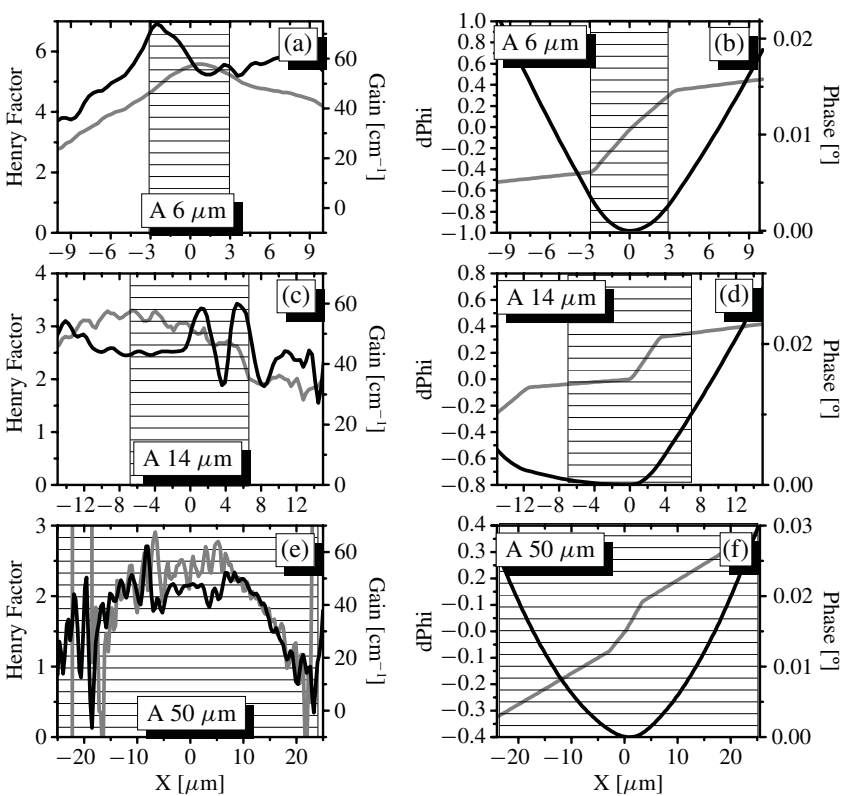

Fig. 6. Lateral Henry parameter (grey curves) and the lateral gain (black curves) profiles [(a), (c), and (e)], relative phase (black curves) and its derivative (grey curves) [(b), (d), and (f)] for lasers with structure A evaluated at $\mathrm{I}=1.5 \mathrm{I}_{\mathrm{th}}$, for stripe widths of $6 \mu \mathrm{m}$ (top), $14 \mu \mathrm{m}$ (middle) and $50 \mu \mathrm{m}$ (bottom), respectively. The shaded areas indicate the stripe width.

The devices with stripe width between $12 \mu \mathrm{m}$ and $20 \mu \mathrm{m}$ are in a transition range between the two regimes described above. Unlike the case of the wide stripes, the pump profile is not sufficient to sustain the formation of a local waveguide. It is possible that the optical field is driven towards the higher index of the un-pumped regions leading to an unstable competition between the gain-guiding and carrier induced antiguiding. This can then lead to the formation of an asymmetric near-field intensity that is the direct result of a spatial offset between the real and imaginary part of the dielectric constant. In fact, in Fig. 4 (c), it is possible to see the offset between the near-field intensity and the calculated gain profile. This is a consequence the phase profile being asymmetric (Fig. 6(c)) leading to an asymmetry in the far-field intensity with a main lobe dominating.

\section{B. Near- and Far-Field Intensities for Structure B}

Fig. 5 (a), (c) and (e) show the extracted gain curves (grey curves) for the devices of structure B. The deep etch of the p-cladding layer further confines the carriers creating a lateral refractive index profile under the stripe such that it is lower in the pumped region in comparison to structure A-type devices because of the higher carrier density. The refractive index step between the pumped and un-pumped region is higher and steeper compared to that of the devices of structure A. Passive waveguide simulations show that there is no builtin positive step in the refractive index because of the lateral etching. Hence our method of phase-profile extraction still remains valid.

The near-field intensity for the $6 \mu \mathrm{m}$ device is single-lobed with a shoulder on the left of the main lobe. This shoulder is due to the additional peak of the gain, visible in Fig. 5(a). For 
this device the main lobe is gain guided, as shown in the black curve in Fig. 5(a). The off-centre near-field intensity is due to a strong anti-guiding effect at the centre of the pumped region with the field drifting to one side driven by spatial noise in the lateral pump profile.

The $14 \mu \mathrm{m}$ wide device is characterized by a near-field intensity with a second order mode-like pattern that has a mixed filamentary and gain guided nature (see Fig. 5 (c)). The device starts with a second order-like mode which becomes filamentary immediately above threshold. The $14 \mu \mathrm{m}$ case also shows the corresponding lateral gain profile (calculated) almost counter-correlated to the near-field intensity. This indicates that the anti-guiding is weaker in the $14 \mu \mathrm{m}$ case in comparison to the $6 \mu \mathrm{m}$ case (similar to the structure A devices) resulting in a predominantly gain-guided mode. However the anti-guiding enough to steer the central lobe away from $x=0$. The far-field, shown in Fig. 5(d) is double lobed with the lobes centered at the same angle and with one stronger that the other. The angular position is a mark of the multimodal character of the near-field, while the asymmetry is due to the noise present in the pump profile [20].

The $50 \mu \mathrm{m}$-wide stripe because of the limited current spreading shows a near-field intensity that is double-lobed in the center of the stripe with two intense peaks at the edges of the stripe (Fig. 5(e)). Even in this case there a countercorrelation between the calculated lateral gain profile and the near-field intensity is noticeable. Since the lateral gain profile has sharp peaks just inside the stripe edges, the corresponding lateral refractive index will have sharp dips at the same spatial locations. This causes the field to be anti-guided and diffract laterally outside the stripe. The field however decays quickly as there are very few carriers to sustain it outside the pumped region. The lateral peaks in the near-field intensity (also known as bat-ears, [19]) are as a result of the excess gain and are not due to the self-focusing. In fact, these sharp peaks are located at the boundary between the pumped and un-pumped regions where a relatively high step in the refractive index is present. Similar argument can be made to explain the nearfield intensity structure directly under the stripe. If there is strong anti-guiding at the centre of the stripe, then the field will pushed towards the edge and self-focusing balanced by diffraction can create the near-field structure as observed, with two lobes centered to $\mathrm{x} \pm 15 \mu \mathrm{m}$.

The far-field intensity has a central double lobed peak and lateral side peaks. The double-peaked main lobe is the result of the filamentary mode formation while lateral side lobes are due Fourier transform of the bat-ears. The behavior of this device clearly shows that the near-field intensity is made of two components: one of filamentary origin and the other one originating from an excess gain at the edges of the pumped region. Experimentally it was not possible to focus the nearfield of this device to a single lobe. This clearly indicated that the near-field is not spatially coherent.

\section{Henry Parameter and Phase Front Lateral Profile}

The calculated and measured far-field intensities match very closely for the narrower stripes using the iterative procedure outlined at the end of section II suggesting that the extracted phase profile is reliable. On the other hand, because of its intrinsic simplicity, the match for the wider stripe devices is not as good. In those cases the presence of higher order modes perturbs the phase profile with small ripples that are responsible of some particular features in the measured farfield intensity. These features are smoothed in the model used here. The resulting phase-profiles are quasi-parabolic having different degrees of asymmetry depending on the individual device (see Fig. 6, black curves). The asymmetry in the phase leads to an asymmetric far-field intensity profile, even when the near field profile is symmetric, and vice versa. However, as shown below the phase profile asymmetry is a consequence of a non-linear interaction between the gain and the photon density, so if the gain function is laterally asymmetric then the photon density profile will be asymmetric too. An asymmetric phase profile is not possible without an asymmetry in the lateral gain and/or in lateral photon-density profile.

From (1), the phase derivative is given by:

$$
\phi^{\prime}(x)= \pm \sqrt{\left[\gamma^{\prime}(x)\right]^{2}-\gamma^{\prime \prime}(x)+k_{0} n_{b} \alpha_{H}(x) \Gamma_{y} g(x)} .
$$

The phase derivative represents the local angle of the phase front at the mirror facet. Choice of the sign in (12) flips the farfield laterally. When the phase profile has a perfect V-shape, its derivative is sigma-shaped (see Fig. 6 (b), (d) and (f)) and quasi-linear under the effective pumped stripe (given by a bellshaped gain profile) reaching its extreme values outside the stripe. If the gain profile has local minimum then the phase derivative has a central linear region characterized by different slopes.

The gain and Henry parameter lateral profiles are mutually dependent lateral functions, so $\alpha_{H}$ cannot be considered as a constant as already theoretically shown by other authors [21]. As shown in Figs. 6 and 7, for both structure A and B the Henry parameter maximum close to the center of the stripe and attains minima values at the boundaries of the effective stripe width. The maximum is due to the high carrier concentration that provokes a negative variation of the refractive index combined to the lower local derivative of the gain with the carriers below the stripe due to the gain clamping. In the regions outside the stripe the carrier density is lower and diminishes monotonically moving far from the pumped region until is null. Here the refractive index variation is minimal due to low carrier concentration while the gain is not clamped and its relative variation with the current is then higher thus minimizing $\alpha_{H}$. The extracted Henry parameter profiles are reliable inside the effective widths of the pumped region in the devices considered, where the influence of the discontinuities in the near field intensity-derivative profiles is less. For both $\mathrm{A}$ and $\mathrm{B}$ type structures, $\alpha_{H}$ profiles are correlated to the respective gain profiles, resembling their shapes. In the case of structure A devices, the magnitude of $\alpha_{H}$ is inversely proportional to the laser stripe width. This is related to the current density which is larger for the narrowest stripes. This behavior is not evident in the structure B devices. 

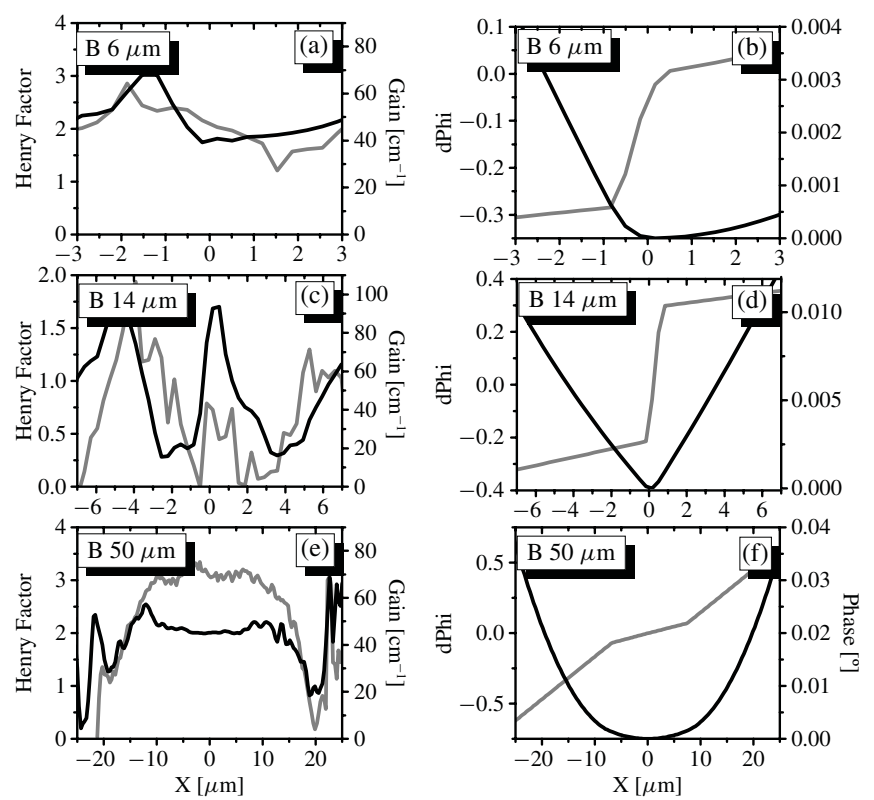

Fig. 7. Lateral Henry parameter (grey curves) and the lateral gain (black curves) profiles [(a), (c), and (e)], relative phase (black curves) and its derivative (grey curves) [(b), (d), and (f)] for lasers with structure B evaluated at $\mathrm{I}=1.5 \mathrm{I}_{t h}$, for stripe widths of $6 \mu \mathrm{m}$ (top), $14 \mu \mathrm{m}$ (middle) and $50 \mu \mathrm{m}$ (bottom), respectively.

Devices with higher asymmetry in the near- and far-field intensities such as the $14 \mu \mathrm{m}$ wide structure A device and $6 \mu \mathrm{m}$ wide structure B devices also have a strong asymmetry in the calculated phase profile (see Fig. 6 (b) and (c)). All the other devices for both structures types $\mathrm{A}$ and $\mathrm{B}$ are characterized by reasonably symmetric convex phase profiles with the sharpness inversely proportional to the stripe width and the etch depth. So devices with wider stripe widths and a deeper etch are likely to show multi-peaked narrower far-field intensities, while narrow stripe devices will provide broader far-fields with an intensity pattern similar to the near-field intensity

\section{CONClusion}

We introduced a model based on the scalar Helmholtz equation that provides a method to estimate the above threshold lateral profiles for the Henry parameter and the gain through recovery of the lateral near-field phase profile. The phase profile is obtained simply by using the near- and farfield intensities measured from each device. The model explains the experimental characteristics obtained on gain-guided laser structures with different levels of current spreading. We showed that for lasers with significant lateral current spreading the near- and far-field intensity patterns depend on the stripe width. All the Henry parameter profiles show their maxima located under or in the vicinity of the laser pumped stripes. The method is more successful for narrow stripe lasers with large current spreading levels.

We distinguished three regimes of operation for lasers with significant lateral current spreading depending on the contact stripe width. Due to the large lateral current spreading, the refractive index profile is broad with a smooth variation between its value inside the stripe and outside the stripe. For $6 \mu \mathrm{m}-$ $10 \mu \mathrm{m}$ wide stripe lasers the gain-photon interaction results in a strong anti-guiding effect, resulting in a double-lobed near and far fields. The $12-20 \mu \mathrm{m}$ wide stripe lasers present unstable characteristics as a result of competition between gain-guiding and anti-guiding. For those devices in particular, the non-linear gain-photon interaction leads to an asymmetric lateral phase profile, responsible for the asymmetries in the near- and far-field intensities. For the $30-50 \mu \mathrm{m}$ wide stripe lasers a broad gain and slow change in the refractive index at the boundaries between the pumped and un-pumped regions lead to a formation of a local waveguide which supports higher order modes.

Because of the weak current spreading and the high confinement of the carriers in structure B the refractive index discontinuities between the injected and non-injected regions is steeper. For $6-10 \mu \mathrm{m}$ wide stripe lasers gain-guiding is the dominant guiding mechanism. $12-20 \mu \mathrm{m}$ devices are characterized by a near-field intensity with a second order mode-like pattern that have a mixed filamentary and gain guided nature. The near-fields of $30-50 \mu \mathrm{m}$ wide stripe lasers are of filamentary origin with the lateral "bat-ears" being the result of local excess of the gain.

\section{REFERENCES}

[1] C. H. Henry, "Theory of the linewidth of semiconductor lasers," IEEE J. Quantum Electron., vol. 18, no. 2, pp. 259-264, Feb. 1982.

[2] M. Osinski and J. Buus, "Linewidth broadening factor in semiconductor lasers-An overview," IEEE J. Quantum Electron., vol. 23, no. 1, pp. 9-29, Jan. 1987.

[3] D. Rodríguez, I. Esquivias, S. Deubert, J. P. Reithmaier, A. Forchel, M. Krakowski, M. Calligaro, and O. Parillaud, "Gain, index variation, and linewidth-enhancement factor in 980-nm quantum-well and quantum-dot lasers," IEEE J. Quantum Electron., vol. 41, no. 2, pp. 117-126, Feb. 2005.

[4] D. Byrne, W. H. Guo, R. Phelan, Q. Y. Lu, J. F. Donegan, and B. Corbett, "Measurement of linewidth enhancement factors for InGaAlAs laser diode by Fourier series expansion method," Electron. Lett., vol. 43, no. 21, pp. 1145-1145, Oct. 2007.

[5] G. P. Agrawal, W. B. Joyce, R. W. Dixon, and M. Lax, "Beampropagation analysis of stripe-geometry semiconductor lasers: Threshold behavior," Appl. Phys. Lett., vol. 43, no. 1, pp. 11-13, Jul. 1983.

[6] R. J. Lang, A. G. Larsson, and J. G. Cody, "Lateral modes of broad area semiconductor lasers: Theory and experiment," IEEE J. Quantum Electron., vol. 27, no. 3, pp. 312-320, Mar. 1991.

[7] M. R. Matthews, R. B. Dyott, and W. P. Carling, "Filaments as optical waveguides in gallium-arsenide lasers," Electron. Lett., vol. 8, no. 23, pp. 570-572, Nov. 1972.

[8] J. R. Marciante and G. P. Agrawal, "Nonlinear mechanisms of filamentation in broad-area semiconductor lasers," IEEE J. Quantum Electron., vol. 32, no. 4, pp. 590-596, Apr. 1996.

[9] J. R. Marciante and G. P. Agrawal, "Spatio-temporal characteristics of filamentation in broad-area semiconductor lasers," IEEE J. Quantum Electron., vol. 33, no. 7, pp. 1174-1179, Jul. 1997.

[10] G. H. B. Thompson, D. F. Lovelace, and S. E. H. Turley, "Kinks in the light/current characteristics and near field shifts in $(\mathrm{GaAl}) \mathrm{As}$ heterostructure stripe lasers and their explanation by the effect of selffocusing on a built-in optical waveguide," Solid-State Electron Devices, vol. 2, no. 1, pp. 12-30, Jan. 1978.

[11] P. A. Kirkby, A. R. Goodwin, G. H. B. Thompson, and P. R. Selway, "Observations of self-focusing in stripe geometry semiconductor-lasers and development of a comprehensive model of their operation," IEEE J. Quantum Electron., vol. 13, no. 8, pp. 705-719, Aug. 1977.

[12] B. W. Hakki and T. L. Paoli, "Gain spectra in GaAs doubleheterostructure injection lasers, J. Appl. Phys., vol. 46, no. 3, pp. 1299 1306, Mar. 1975. 
[13] D. Mehuys, R. J. Lang, M. Mittelstein, J. Salzman, and A. Yariv, "Self-stabilized nonlinear lateral modes of broad area lasers," IEEE J. Quantum Electron., vol. 23, no. 11, pp. 1909-1919, Nov. 1987.

[14] H. Braun, S. Rogowsky, U. Schwarz, S. Bruninghoff, A. Lell, S. Lutgen, and U. Strauss, "Supermodes in broad area (Al,In)GaN laser diodes," IEEE J. Quantum Electron., vol. 45, no. 9, pp. 1074-1083, Sep. 2009.

[15] K. Petermann, "Some relations for the far field distribution of semiconductor lasers with gain-guiding," Opt. Quantum Electron., vol. 13, no. 4, pp. 323-333, Jul. 1981.

[16] P. Sajewicz, C. Kelleher, and B. Corbett, "Influence of structure on external efficiency of wide stripe semiconductor lasers based on the analysis of spontaneous emission," IET Optoelectron., vol. 4, no. 4, pp. 149-158, Aug. 2010.

[17] J. Houlihan, J. R. O’Callaghan, V. Voignier, G. Huyet, and J. G. McInerney, "Experimental observation of traveling waves in the transverse section of a laser," Opt. Lett., vol. 26, no. 20, pp. 1556-1558, Oct. 2001.

[18] W. Strifer, "An analytic study of (GaAl)As gain guided lasers at threshold," IEEE J. Quantum Electron., vol. 18, no. 5, pp. 856-864, May 1982

[19] J. K. White, J. G. McInerney, and J. V. Moloney, "Formation of sharply peaked sidelobes in large aperture single-pass laser amplifiers," Electron. Lett., vol. 31, no. 1, pp. 38-39, Jan. 1995.

[20] P. K. Jakobsen, J. V. Moloney, A. C. Newell, and R. Indik, " Space-time dynamics of wide-gain-section lasers," Phys. Rev. A, vol. 45, no. 11, pp. 8129-8137, Jun. 1992.

[21] E. Gehrig, O. Hess, "Dynamic amplitude-phase coupling in quantumdot lasers," Appl. Phys. Lett., vol. 86, no. 20, pp. 203116-1-203116-3, May 2005.

[22] N. Bleistein and R. Handelsman, Asymptotic Expansions of Integrals. New York: Dover, 1975.

Roberto Pagano (S'10) received the B.Sc. and M.Sc. (hons.) degrees in electronics engineering from the Politecnico di Bari, Bari, Italy, in 2004 and 2007, respectively. He is currently working toward the Ph.D. degree in physics.

He joined the III-V Materials and Devices Group, Tyndall National Institute, Cork, Ireland, in 2007. He has also worked as an invited student at the Universidad Politécnica de Madrid, Madrid, Spain, in 2008, and at the Max Born Institut, Berlin, Germany, in 2009. His current research interests include high power lasers, covering the design and the test of the devices, particularly the difference between quantum dot and quantum well based active media on the global performances of the device, as the efficiency, the beam quality and the reliability.
Jayanta Mukherjee received the B.Sc. (hons.) and M.Sc. degrees in physics from the University of Delhi, Delhi, India, in 2000 and 2002, respectively. He received the M.Tech. degree in lasers from Devi Ahilya University, Indore, India, in 2004. In 2005, he began Ph.D. research at the Optoelectronics Group, Department of Physics, Tyndall National Institute, University College Cork, Cork, Ireland, on the topic of power and spatial coherence scaling in wide aperture semiconductor lasers. He received the Ph.D. degree from the same group in June 2009.

He joined the Photonics Group, Advanced Technology Institute, University of Surrey, Guildford, U.K., after brief post-doctoral work in the Optoelectronics Group. He is currently a Research Fellow at the Photonics Group, Advanced Technology Institute, University of Surrey, on a project aiming at laser power beaming from space. He has worked as an Invited Researcher at the College of Optical Sciences, University of Arizona, Tucson, and at the Max Born Institute, Berlin, Germany, in 2006 and 2007, respectively, for four months each. He was also an Assistant Lecturer with the Applied Mathematics Department, University College Cork, from 2006 to 2009. His current research interests include cavity nonlinear optics, laser power converters, effect of injected coherence and spatiotemporal dynamics in quantum dot lasers, vertical-external-cavity surface-emitting-lasers, and thermal management and package designing for semiconductor lasers.

Pawel Sajewicz received the M.Sc. degree from Warsaw University of Technology, Warsaw, Poland, in 2000. He received the Ph.D. degree from Tyndall National Institute, University College Cork, Cork, Ireland, in March 2010.

He was a Junior Researcher at the Institute of Electron Technology, Warsaw, from 2000 to 2004. In 2005, he moved to the III-V Materials and Devices Group, Photonics Research Center Tyndall National Institute, Cork, as a Ph.D. student. His current research interests include optical and electrical characterization of high power, infrared and red emitting edge emitting lasers, influence of carrier loss mechanisms on the internal efficiency, and beam quality of these devices based on experimental methods using analysis of spontaneous emission spectra.

Brian Corbett received the B.A. Mod degree in experimental physics and mathematics and the M.Sc. degree in theoretical physics from Trinity College Dublin, Dublin, Ireland, in 1982 and 1984, respectively.

He has been a Scientist at Tyndall National Institute, University College Cork, Cork, Ireland, researching the physics and technology of III-V based light emitting semiconductors used in communications, sensing, and lighting applications, since 1990. His current research interests include use of structuring in order to integrate different optical functions on a single chip. 